\title{
ArcheoSciences
}

Revue d'archéométrie

$30 \mid 2006$

Varia

\section{Réflexions sur l'organisation technico-sociale des activités de forge à La Tène finale dans l'Aisne}

Thoughts on the technico-social organization of the forging activities at the final La Tène period in the Aisne department

Sylvain Bauvais et Philippe Fluzin

(2) OpenEdition

Journals

Édition électronique

URL : https://journals.openedition.org/archeosciences/123

DOI : 10.4000/archeosciences. 123

ISBN : 978-2-7535-1595-6

ISSN : 2104-3728

Éditeur

Presses universitaires de Rennes

Édition imprimée

Date de publication : 31 décembre 2006

Pagination : 25-43

ISBN : 978-2-7535-0456-1

ISSN : $1960-1360$

Référence électronique

Sylvain Bauvais et Philippe Fluzin, « Réflexions sur l'organisation technico-sociale des activités de forge à La Tène finale dans l'Aisne », ArcheoSciences [En ligne], 30 | 2006, document 3, mis en ligne le 31 décembre 2008, consulté le 01 février 2022. URL : http://journals.openedition.org/archeosciences/ 123 ; DOI : https://doi.org/10.4000/archeosciences.123

Article L.111-1 du Code de la propriété intellectuelle. 


\title{
Réflexions sur l'organisation technico-sociale des activités de forge à La Tène finale dans l'Aisne
}

\author{
Sylvain Bauvais* et Philippe Fluzin**
}

\begin{abstract}
Résumé : Le Nord du Bassin parisien est caractérisé par une absence de sites de réduction du minerai de fer pour La Tène finale, voire pour toute l'antiquité. Toutefois, les activités de forge sont omniprésentes pour la fin de l'âge du Fer. Cette zone est en revanche cernée par plusieurs régions productrices de fer brut.

Dans ce contexte, l'acquisition de la matière première, sous la forme de demi-produits, semble donc astreinte à un approvisionnement à moyenne ou longue distance. Les activités de forge doivent alors s'organiser selon un schéma particulier, propre à l'organisation socio-économique de cette période dans cette région.

À travers l'analyse des demi-produits de la région et de la chaîne opératoire de post-réduction (forge) de 5 sites de l'Aisne, nous allons tenter de préciser les hypothèses et les questionnements sur la circulation des demi-produits et donc sur l'organisation régionale de la production d'objets en fer.

Jusqu’à présent, dans le nord du Bassin parisien, les indices clairs permettant de caractériser la matière première travaillée sur les sites restaient rares. Récemment, plus de 500 demi-produits d'une morphologie particulière (que nous appellerons barres à douille) ont pu être identifiés. Toutefois, la matière première circulait sous de multiples formes et les déchets engendrés par leur transformation peuvent apporter un complément nous permettant d'aborder ce point crucial pour la compréhension de l'organisation de cette production. La quantification des pertes en parties retrouvées dans ces culots de forge ainsi que la propreté inclusionnaire de celles-ci, corrélée à celles des demi-produits et des objets, permettent de donner une idée de la qualité du métal travaillé.

Suite à ces analyses, il est possible de classer schématiquement ces demi-produits, selon leur homogénéité inclusionnaire, en trois catégories : une qualité très médiocre proche de la masse brute, une mauvaise qualité et une bonne qualité inclusionnaire.

Les résultats acquis jusqu'à présent permettent d'entrevoir une organisation complexe des activités de forge dont les principaux modes nous restent encore majoritairement inconnus.

On remarque, dans cette région, en ce qui concerne les activités sidérurgiques, un croisement constant entre des impératifs d'ordre géographiques, technologiques et socio-économiques.
\end{abstract}

Abstract: The North of the Parisian Basin is characterized by an absence of iron ore reduction sites throughout the final La Tène period as well as for the whole antiquity. Nevertheless, the smithing activities are omnipresent throughout the end of the Iron Age. This area is on the other hand surrounded by several productive brute iron areas.

In this context, the acquisition of raw material, under the form of semi-finished products, seems therefore to depend on a provisioning from medium or long distance.

The smithing activities must then be organized according to a particular outline, linked to the socio-economic organization of this period in this region. Through the analyses of the semi-finished products of this region and the post-reduction operating system (smithing) of 5 sites of the Aisne department, we will attempt to specify the hypotheses and the questionments on the semi-finished products circulation and therefore on the regional production organization of iron objects.

So far, in the North of the Parisian Basin, the clear clues allowing a characterisation of the raw material worked on the sites remained rare. Recently, about 250 half-products of particular morphology (that we will call "barres à douille") could be identified.

* Doctorant, UMR 5060 du CNRS, laboratoire "Métallurgie et Cultures", Université de Technologie de Belfort-Montbéliard, 90010 Belfort Cedex, France. (sylvain.bauvais@utbm.fr)

** Directeur de recherche, UMR 5060 du CNRS, laboratoire "Métallurgie et Cultures ", Université de Technologie de Belfort-Montbéliard, 90010 Belfort Cedex, France. (philippe.fuzin@utbm.fr) 
However, the raw material circulated under multiple forms and the waste generated by their transformation can bring a complement enabling us to approach this crucial point for the comprehension of this production organization. The quantification of the losses found in these smithing cakes as well as the inclusional cleanliness of those, correlated with those of the semi-finished products and objects, allows to give an idea of the quality of the worked metal.

Following these analyses, it is possible to classify schematically these semi-finished products, according to their inclusional homogeneity, in 3 categories: a very mediocre quality near to the brute mass, a bad quality and a good inclusional quality. The results obtained until now make it possible to foresee a complex organization of the smithing activities whose principal modes still remain mainly unknown to us.

We can note, in this region, with regards to the ironworking activities, a constant crossing between imperatives of geographical, technological and socioeconomical orders.

Mots clés : La Tène, Bassin parisien, sidérurgie ancienne, forge, scorie, analyses métallographiques.

Key words: La Tène period, Parisian Basin, early ironworking, smithing activity, slag, metallurgical analysis.

\section{INTRODUCTION}

\subsection{Contexte archéologique}

Les études archéologiques dans la vallée de l'Aisne et ses environs connaissent depuis une trentaine d'années un suivi soutenu et constant. Tous les matériaux des époques protohistoriques et les systèmes socio-économiques les structurant ont fait l'objet de travaux universitaires ou de programmes de recherche de l'ERA 12 puis du laboratoire Protohistoire Européenne de l'UMR 7041 du CNRS et seule la métallurgie du fer n’avait pas encore été abordée de façon systématique.

Les résultats de cette réflexion sur l'organisation technicosociale des activités de forge à La Tène finale dans l'Aisne s'intègrent donc dans un ensemble fourni de données sur cette période, dans cette région.

Le nord du Bassin parisien est caractérisé par une absence de sites de production primaire du fer à partir du minerai (réduction) pour La Tène finale voire pour toute l'antiquité. Cette zone est, en revanche, cernée par plusieurs régions productrices de fer brut :

- Au Sud, les sites sénons (Yonne), comme Les Clérimois (Dunikowsky 1995; Dunikowsky, Cabboï 1995), sont les plus connus et leur production semble être la plus intense (Fig. 1A). Le nord de la Seine-et-Marne renferme également des indices importants de réduction, mais pas avant le Haut Moyen Âge, comme les sites de Vert-Saint-Denis " les Fourneaux " (Daveau, Goustard 2000), Roissy-en-Brie "les Grands Champs " et " la Ferme Sassinot " (Lorcquet à paraître) (Fig. 1B). Faut-il y voir une découverte tardive des minerais locaux ou une lacune de la recherche? Les travaux réalisés en ce moment par l'INRAP dans le secteur permettront rapidement d'avoir une vision plus claire sur ces questions.

- À l'Ouest, les fouilles réalisées sur le tracé de l'autoroute A28 par Sandra Cabboï révèlent une activité intense, de l'extraction à la réduction du minerai de fer pour la fin de l'âge du Fer (Dunikowski, Cabboi 2001) (Fig. 1C). Plus au Nord, le pays de Bray recèle également des indices de réduction au cours de La Tène finale sur le site du fossé « les prés de Montadet " (Colliou à paraitre) (Fig. 1D).

- À l'Est, les Ardennes et le district entre Sambre-et-Meuse présentent de nombreux gisements de minerai et de sites de réduction mais aucun n'est connu jusqu'à présent pour l'âge du Fer (Fig. 1E et F).

Dans ce contexte, l'acquisition de la matière première sous la forme de demi-produits semble astreinte à un approvisionnement à moyenne ou longue distance.

Les activités de forge (transformation mécanique et chimique du métal dans le but de produire un objet), omniprésentes au cours de la fin de l'âge du Fer (Fig. 1), doivent alors s'organiser selon un schéma particulier, propre à l'organisation socio-économique de cette période dans cette région. On note en effet à partir de La Tène finale un «bruit de fond " sidérurgique sur l'ensemble des sites. Cela se matérialise par la présence systématique de quelques scories correspondant à une simple activité domestique d'entretien des outils (agricoles, culinaire...) et parfois par des concentrations de 1 à $100 \mathrm{~kg}$ qui peuvent être rattachées à de réelles activités artisanales.

Ces activités réclament l'acquisition d'une matière première plus ou moins brute (demi-produits) dont l'état est déterminé par la vocation de la production et par la fonction $\mathrm{du}$ site producteur.

Pour cette période (Fig. 2), 15 sites d'atelier sont connus dans l'Aisne et la moyenne vallée de l'Oise (Fig. 1). 


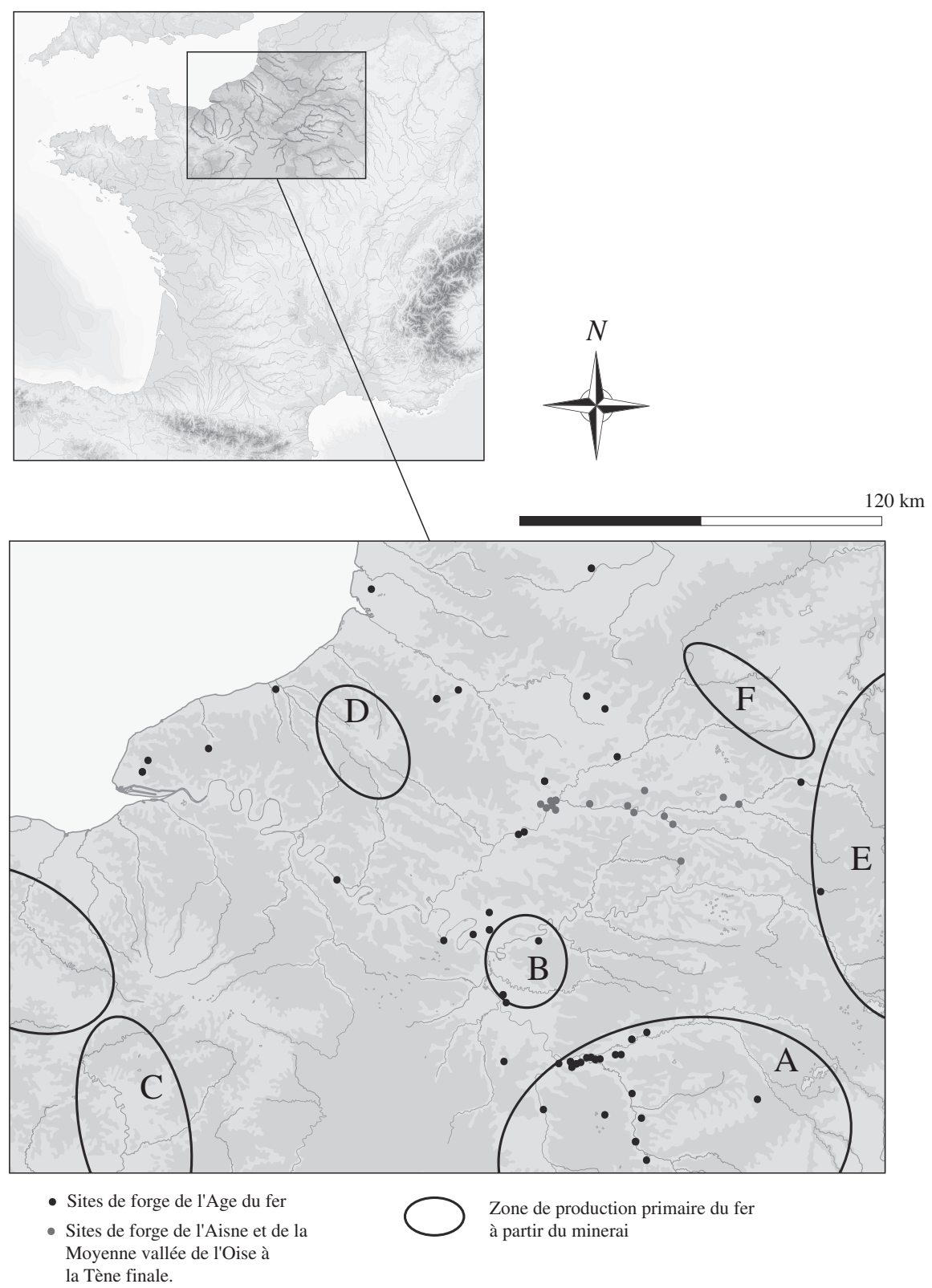

Figure 1 : Cartes de répartition des indices sidérurgiques de l'Âge du fer dans le Nord du Bassin parisien et des zones connues de réduction primaire du minerai.

Figure 1: Distribution map of the steel-making Iron Age indications in the North of Paris Basin and zones known for primary reduction of the ore.

À travers l'analyse des demi-produits de la région et de la chaîne opératoire de post-réduction (forge) de 5 sites de l'Aisne, nous allons tenter de préciser les hypothèses et les questionnements sur la circulation des demi-produits et par conséquent sur l'organisation régionale de la production d'objets en fer.

\subsection{Méthodologie d'étude}

Afin de caractériser le métal arrivant sur chacun des sites, nous avons sélectionné en priorité les fragments de demiproduits, les chutes de forge ainsi que les culots comportant une grande quantité de métal. En ce qui concerne les culots, seule la moins bonne qualité inclusionnaire du métal retrou- 


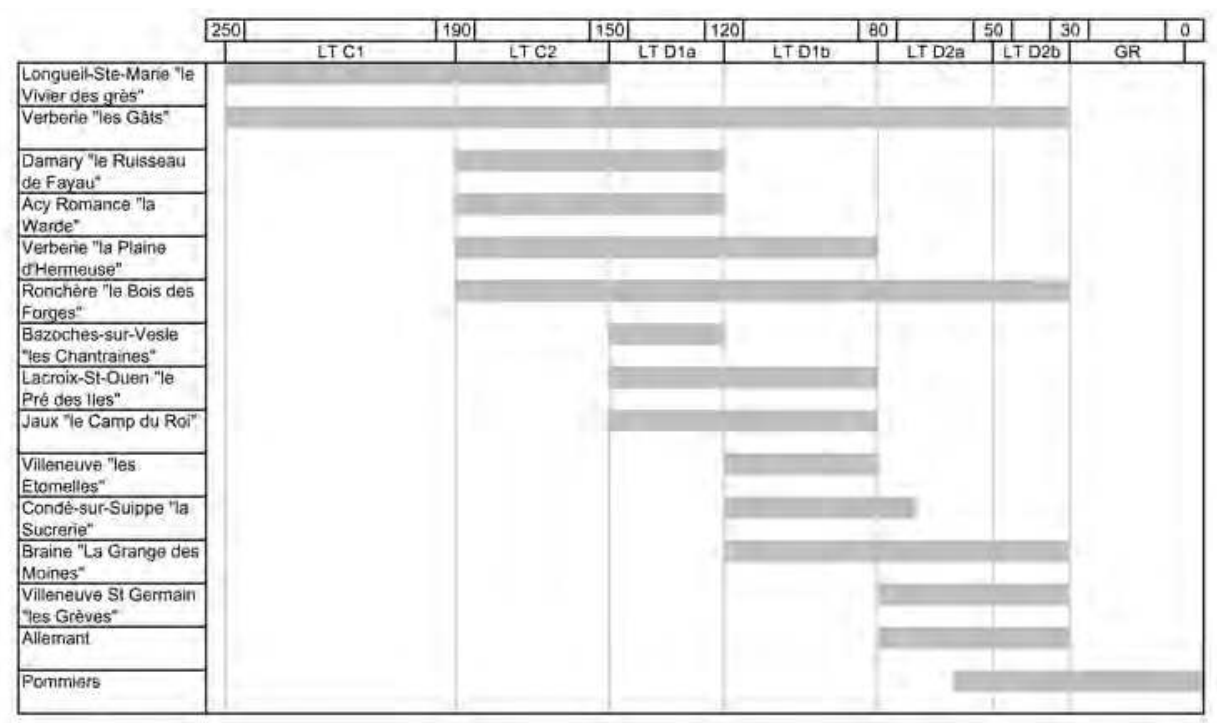

Figure 2 : Chronologie des sites de La Tène finale dans l'Aisne et dans la moyenne vallée de l'Oise.

Figure 2: Chronology of the final La Tène sites in the Aisne and in the average Oise river valley.

vée nous permet de caractériser le demi-produit travaillé. En effet, le reste peut correspondre à une phase de travail postérieure, faisant suite aux premiers martelages. Pour les mêmes raisons, les objets finis qui résultent d'un travail en forge et donc d'une épuration liée au martelage de mise en forme ne seront pas présentés dans cette étude.

Pour chacun des sites, les culots de forge ont fait l'objet d'un premier tri par famille morphologique (Mangin et al. 2000a et b; Anderson et al. 2003). Ensuite 2 ou 3 échantillons par famille ont été sélectionnés pour analyse, a priori considérés comme représentatifs de leur groupe morphologique. Les échantillons de cette étude ont été analysés au microscope métallographique afin de déterminer leur structure et leur composition. L'étude de chaque échantillon respecte un protocole rigoureux, et qui se décompose comme suit (MANGIN et al., 2000a et b).

- Description morphologique externe et métrologie (dimension);

- Pesée;

- Prise de vue photographique de dessus et de dessous (voire de côté s'il y a lieu);

- Tronçonnage sous lubrifiant (en 1 ou plusieurs points);

- Photographie du ou des plans de coupe. Ces clichés faciliteront d'une part l'observation macrographique (analyse d'image) de l'échantillon (hétérogénéité, stratification, porosité, répartition des différents composants...), et d'autre part le repérage et le positionnement des examens micrographiques qui seront reportés sur le dessin de la section;
- Dessin des plans de coupes pour permettre le report des références aux micrographies;

- Enrobage dans une résine thermodurcissante appropriée;

- Polissage mécanique sous lubrifiant jusqu'à une granulométrie de $1 \mu \mathrm{m}$;

- Observation intégrale sous microscope métallographique (grossissement variant de 25 à 1000) et prise de micrographies;

- Attaque chimique au Nital (3\% d'acide Nitrique dans l'alcool);

- Nouvelle observation intégrale au microscope avec micrographie des structures caractéristiques;

- Évaluation des microduretés si nécessaire.

Ensuite, un traitement d'image permet de quantifier les proportions d'éléments (métal, fayalite, argilo-sableux, porosités...) sur la surface de la coupe.

De la même manière, le taux d'inclusions et de porosité dans le métal est recherché et sera regroupé sous le terme de propreté inclusionnaire.

\section{LES DEMI-PRODUITS}

\subsection{Définition d'un demi-produit}

Les activités de post-réduction englobent toutes les phases de travail du métal, dès la sortie du bas fourneau de la masse brute de fer jusqu'à l'élaboration de produits finis (objets) et leur recyclage. 
Ce travail du métal est divisible en différentes séquences, bien que celles-ci ne correspondent pas forcément à une séparation spatio-temporelle. On peut ainsi schématiquement séparer les activités d'épuration-compactage du métal, des activités d'élaboration d'objets.

La masse brute de métal comporte à son état initial une quantité plus ou moins importante d'inclusions de scorie et de charbon de bois. Ces inclusions doivent être éliminées par suage et cinglage de la masse dans un foyer et sur une enclume (celle-ci peut être en bois, en pierre ou en métal - Fluzin 2002). Cette masse comporte également des porosités issues de la réduction mais aussi de l'écoulement de la scorie, qui doivent être écrasées et soudées sur elles-mêmes afin d'obtenir une masse compacte. Cette phase d'épuration-compactage s'opère dans un premier temps par des chauffes et martelages plus ou moins poussés, puis éventuellement par étirement et replis successifs du métal (corroyage), jusqu'à ce qu'il réponde aux exigences techniques réclamées par l'artisan. Cette technique a également pour fonction d'homogénéiser le métal, très hétérogène à la base, dans sa texture et sa composition.

L'épuration peut se réaliser dans un premier temps dès la sortie du bas fourneau, en utilisant l'inertie thermique de la masse brute (Fluzin 2002). Toutefois, un travail d'épuration plus poussé s'effectue a priori dans un foyer ou un four spécifique. Pour ce qui est des phases finales de cette épuration, elle a lieu au cours de la mise en forme de l'objet et se déroule donc dans le foyer de forge. On remarque bien ici la difficulté d'interprétation des différentes structures de combustion et des phases de travail qui leur sont rattachées, sachant surtout que leur morphologie n'est pas forcément très éloignée. L'étude des déchets associés permet de faciliter leur distinction.

À partir de cette masse compacte, il est possible d'appliquer les différentes déformations plastiques que réclame l'élaboration d'objet. La mise en forme du métal englobe un panel de techniques très large que chaque artisan, de chaque période, va mettre en ouvre afin d'obtenir les produits voulus dans son contexte socio-économique.

Cette séparation entre épuration-compactage et élaboration n'a de sens que dans un schéma technique. En réalité, la chaîne opératoire peut être beaucoup plus morcelée. Les interruptions de séquences peuvent intervenir à différents stades, matérialisés par des demi-produits. Un demi-produit concrétise, en effet, un état intermédiaire de la matière entre le métal brut à la sortie du bas fourneau et l'objet fini. Comme nous l'avons précisé, la chaîne opératoire de postréduction peut être très morcelée dans l'espace et dans le temps. Chacune de ces ruptures va engendrer un demi-produit particulier qui peut avoir divers degrés de compaction et de préformage (ébauches).
La taille et la morphologie de ces demi-produits varient en fonction des masses brutes de fer initiales, de la quantité de travail d'épuration réalisée, de la nature du métal (fer/acier) et de la destination du produit. Ceci se traduit de fait par une valeur marchande plus ou moins graduée. C'est donc sous de multiples formes que le métal va circuler et être commercialisé.

Un demi-produit est destiné à la transformation, au stockage et aux échanges. Les différents contextes de découverte de ces demi-produits vont bien dans ce sens. Ils peuvent provenir de rejets d'ateliers, sous la forme de fragments découpés à chaud (transformation), de lieux de stockage ou " caches » de métallurgiste (dépôt de Durrenentzen - Bas Rhin) ou de pertes accidentelles lors de transports commerciaux (Épaves des Saintes-Maries-de-la-Mer - Bouches-duRhône; dragages de la Saône et de l'Oise). Les découvertes en contexte votif sortent, quant à elles, d'une logique technologique, bien qu'elles attestent de la valeur économique de ces produits.

L'artisan va donc acquérir ce métal sous une forme particulière (morphologie, propreté inclusionnaire, composition fer/carbone) suivant plusieurs critères :

- suivant ses besoins (type d'objet à produire) ;

- suivant ses moyens (l'épuration donne une valeur ajoutée au métal, liée à la quantité de travail réclamé par l'activité);

- suivant ses compétences techniques (l'épuration nécessite un savoir faire certain, surtout s'il s'agit d'un acier fortement carburé);

- Suivant son accès aux différents réseaux d'échanges commerciaux.

\subsection{Les demi-produits du nord du Bassin parisien}

Jusqu’à présent, dans le nord du Bassin parisien, les indices clairs permettant de caractériser la matière première travaillée sur les sites restaient rares. Récemment, plus de 500 demi-produits d'une morphologie particulière (que nous appellerons barres à douille) ont pu être identifiés (Fig. 3). Il s'agit de barres ou de lamelles (suivant leur épaisseur), de 20 à $40 \mathrm{~cm}$ de long et de 1 à $5 \mathrm{~cm}$ de large pour une épaisseur pouvant aller de 3 à $5 \mathrm{~mm}$. Une des extrémités se termine par une douille conique en pointe ouverte vers la barre. L'autre extrémité se termine en spatule lorsque celleci est conservée. Ces objets se rencontrent dans deux types de contexte. Ce sont leurs traitements rituels dans les sanctuaires de l'Oise qui ont permis de les mettre en évidence et de pousser la réflexion sur leur fonction (Woimant 1990). 


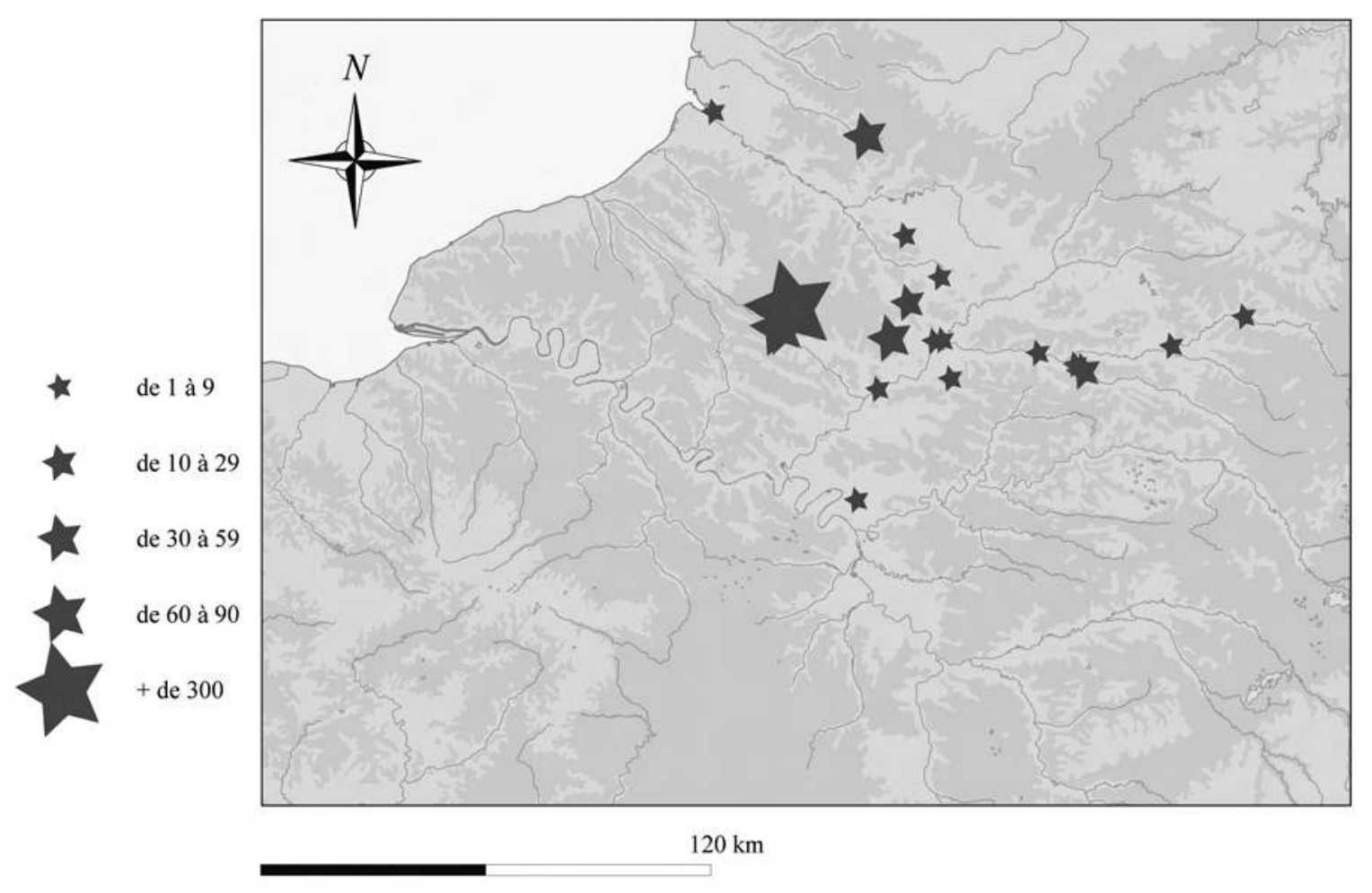

Figure 3 : Répartition des barres à douille dans le Nord du Bassin parisien.

Figure 3: Distribution of the socket-bars in the North of Paris Basin.

Aujourd'hui, plus de 200 individus sont connus sur 7 sites, sacrifiés et déposés dans ces contextes de sanctuaire. Leur présence aux côtés de fragments d'armes, d'objets de parure, d'ossements humains et de pratiques de banquet donne à ce type de demi-produit une valeur autre qu'utilitaire et indique une valeur socio-économique importante.

Toutefois, c'est leur présence en contexte de forge qui a permis de confirmer cette fonction. Vingt-six fragments de ces demi-produits ont, en effet, pu être mis en évidence, entiers ou débités à chaud, dans 11 ateliers de cette région (Fig. 4) (Bauvais 2000; Bauvais, Fluzin 2005).

Leur morphologie particulière, leur relative bonne propreté inclusionnaire ainsi que le caractère local de ces objets semblent plutôt représenter un état final du métal dans ce commerce de matière première.

Dans certaines régions mieux documentées, il est possible de mettre en évidence d'autres types de demi-produits, différents entre eux par leur morphologie et par leur structure interne (propreté inclusionnaire et degrés de compaction).

Ainsi, la matière semble avoir circulé durant cette période sous la forme de loupes mal dégrossies et partiellement compactées (ex : Bourges "Saint-Martin-des-Champs »- Leroy, Merluzzo), ce que l'on retrouve également en éthno-archéologie au Burkina Faso (Fig. 5a), sous la forme de blocs bipyramidaux (ex : dragages de l'Oise) (Fig. 5b), de currency bars (ex : Aulnat - Orengo 2003) (Fig. 5c), de barres à section quadrangulaire (ex : Acy-Romance "la Warde») (Fig. 5d), ainsi que sous la forme de barres à douille (ex : Villeneuve-Saint-Germain « les Etomelles » et Beauvais « les Aulnes du Canada » - Woimant 1990) (Fig. 5e et f).

Cette multiplicité de formes peut avoir plusieurs explications. Soit il s'agit de traditions culturelles différentes (régionale, de " caste " de métallurgistes...), soit, et cela semble plus plausible, elle revêt un caractère technologique. Leurs formes très différentes peuvent être liées au type d'activité dans laquelle elles s'intègrent; sortes de préformes ou d'ébauches des futurs objets. Elles peuvent également se différencier afin de caractériser le degré d'épuration du métal. Ces deux remarques ne s'excluant pas l'une l'autre sachant que, comme nous l'avons déjà dit, selon le type d'objet escompté, la qualité du métal réclamé peut être différente.

Les analyses de ces demi-produits ont permis de caractériser globalement leur degré de compaction, mais toutefois, ces travaux restent encore trop peu nombreux pour tirer des conclusions d'ordre général sur le sujet.

Ainsi, sachant que la matière première circule sous de multiples formes et que les sites de post-réduction renferment rarement des restes de ces demi-produits, les déchets engendrés 


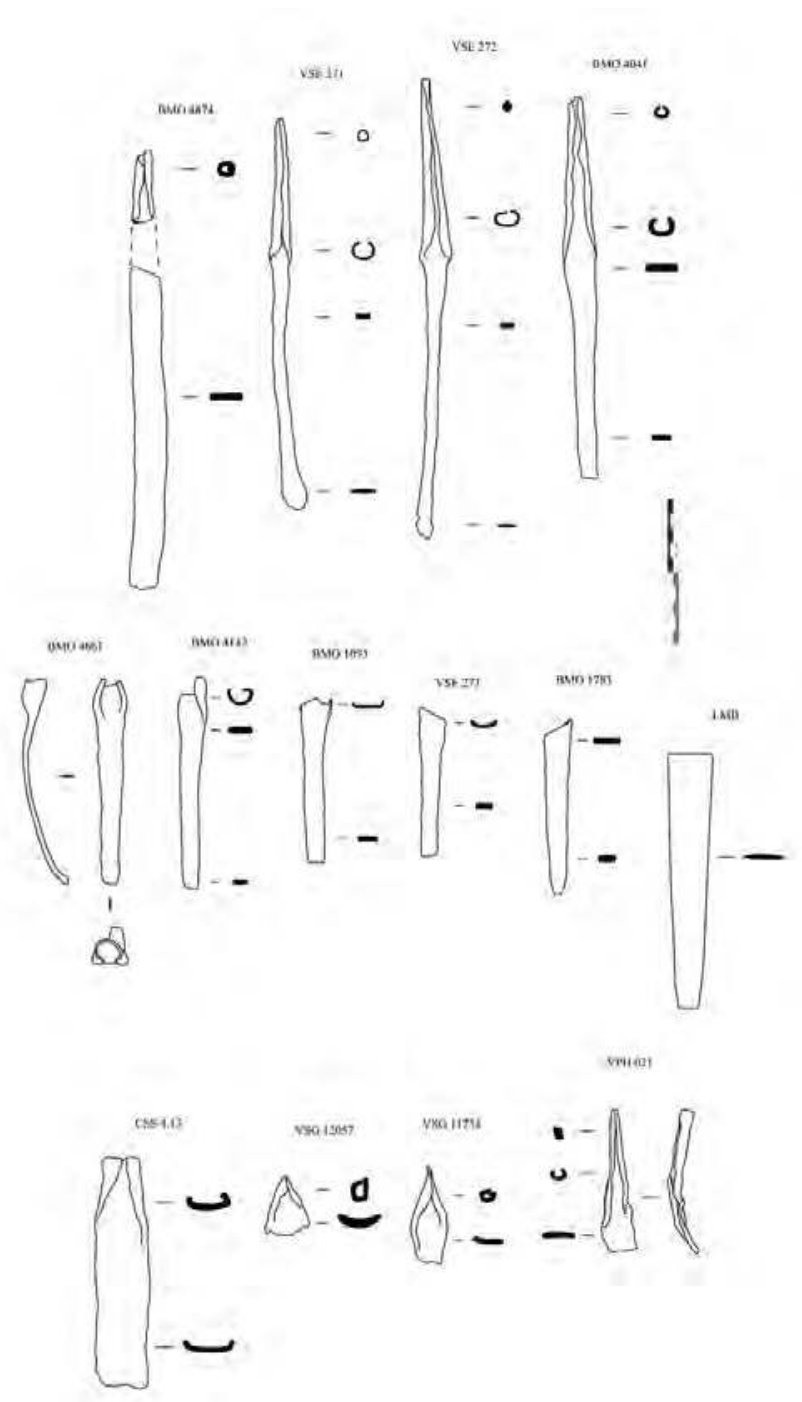

Figure 4 : Barres à douille provenant de contexte d'atelier de l'Aisne et de la moyenne vallée de l'Oise.

Figure 4: Socket-bars from workshop context of the Aisne and the average valley of the Oise river.

par cette activité peuvent apporter un complément nous permettant d'aborder un point crucial pour la compréhension de l'organisation de cette production.

Lors du travail en forge d'une masse de fer, une quantité plus ou moins importante de métal est perdue par l'artisan, autour et dans le foyer de forge (de $80 \%$ de sa masse pour une masse brute de fer dite " sale " à $50 \%$ pour une masse dite " propre ") (Crew 1991; Dillmann et al. 1998; Fluzin 1999; Leroy et al. 2000 ; Fluzin 2002; Fluzin et al. 2004). Ces pertes se retrouvent stratifiées dans les culots. Elles sont plus ou moins importantes suivant la qualité intrinsèque du travail du forgeron (met-il en œuvre tout ce qui est possible pour ne pas perdre son métal?) mais aussi suivant la qualité de compaction du demi-produit.
Les expérimentations ont en effet permis de mettre en évidence une diminution des pertes de métal à mesure que la chaîne opératoire de post-réduction se déroule et donc que la masse métallique s'homogénéise en se densifiant.

La quantification des pertes en partie retrouvées dans ces culots de forge, ainsi que la propreté inclusionnaire de cellesci, corrélée à celles des demi-produits et des objets, permettent de donner une idée de la qualité du métal travaillé.

\section{3. ÉTUDE DE LA QUALITÉ DU MÉTAL TRAVAILlÉ À TRAVERS LES DÉCHETS DE 5 SITES DE FORGE}

Les cinq sites présentés ici se localisent dans le département de l'Aisne. Ils appartiennent tous à une fourchette chronologique allant de La Tène C2 à La Tène D2, soit environ entre 180 et 50/30 avant notre ère (Fig. 2).

\subsection{Condé-sur-Suippe « la Sucrerie »}

Le site est localisé dans la vallée de l'Aisne, à environ $20 \mathrm{~km}$ de la ville de Reims, sur les communes de Condé-surSuippe, Variscourt et Guignicourt (02) (Pion et al. 1997 ; Bauvais 2000; Bauvais, Pion à paraître).

Il s'agit d'une vaste fortification de type oppidum s'étendant sur plus de 170 hectares, installée en fond de vallée sur une vaste terrasse alluviale, dans un méandre de l'Aisne, à la confluence entre cette rivière et la Suippe.

L'occupation s'étend entre le $\mathrm{II}^{\mathrm{e}}$ et le $\mathrm{I}^{\mathrm{er}}$ siècle avant notre ère (la Tène D1b/D2a).

Ce secteur de fouille est centré autour d'un ou plusieurs ateliers de forge, mais contient également des activités de bronziers et de travaux des métaux précieux. La métallurgie est donc l'activité principale de ce quartier.

Les résultats d'analyse :

Le secteur étudié de cet oppidum a livré environ $48 \mathrm{~kg}$ de scorie dont 38 culots de forge entiers. Dix d'entre eux ont fait l'objet d'une étude métallographique. D'autres scories ont été sélectionnées lorsqu'elles présentaient d'importantes pertes de métal.

Un fragment de demi-produit de type barre à douille a également fait l'objet d'une investigation métallographique, ainsi qu'une soie de préhension résultant du repli longitudinal d'une de ces mêmes barres.

L'analyse des culots de forge et des scories métalliques atteste d'un métal de qualité inclusionnaire mauvaise.

Trois culots, représentatifs d'un tiers du corpus total, présentent une forte proportion de perte de métal (entre 5 et $17 \%$ de la surface de la coupe). Ces pertes de métal ont un 


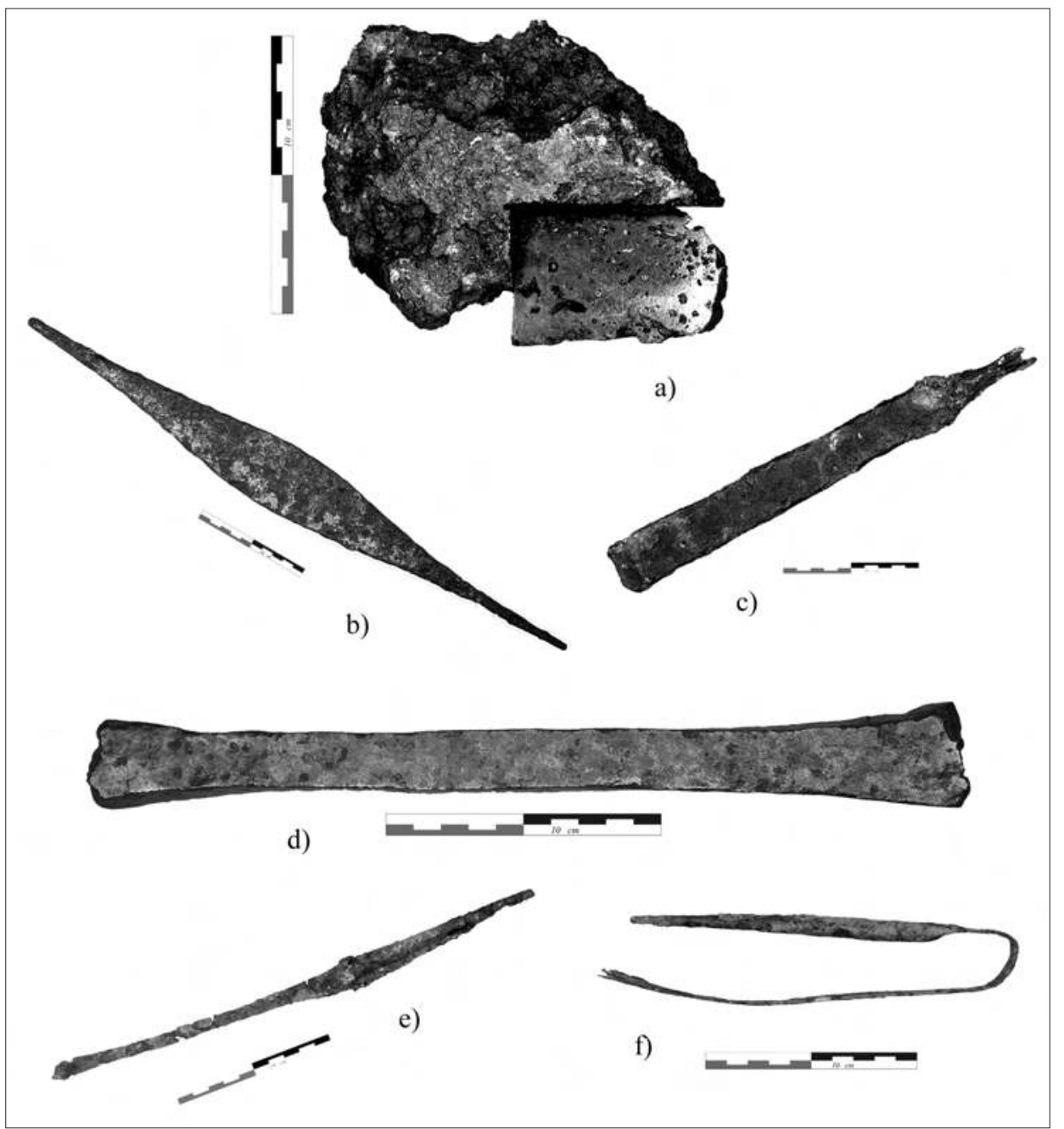

Figure 5 : Présentation non exhaustive de différents types de demi-produits.

Figure 5: Not exhaustive presentation of various types of semi-finished products.

aspect d'éponge (déchiqueté en début de phase) et comportent un grand nombre de porosités plus ou moins vidées de la scorie ainsi que des replis (de 15 à $25 \%$ d'impureté sur la surface analysée du métal). Les impuretés ne présentent pas encore de déformation liée au corroyage. On note par ailleurs sur l'un des échantillons une succession de phases thermiques au fur et à mesure de l'épuration et de la mise en forme (Fig. 6a).

Les scories métalliques, rappelant des " gromps ${ }^{1}$ ", peuvent faire penser à une épuration de masses de fer (ou de fragments) grossièrement compactées. La présence d'un nodule de fonte de 2 à $3 \mathrm{~cm}^{3}$ et d'une masse de $40 \mathrm{~g}$, produit accidentellement lors de la réduction du minerai et qui fut retrouvé en contexte de forge ne peut, notamment,

1. Nosek 1994. pas avoir été aggloméré et transporté dans un demi-produit bien épuré (Fig. 6b). Par ailleurs, la production de fonte en contexte de forge est peu vraisemblable.

L'analyse du mobilier métallique révèle, quant à elle, un métal de mauvaise qualité inclusionnaire. La barre à douille sectionnée à chaud en contexte de forge présente une forte proportion de lignes d'inclusions, majoritairement fayalitiques avec globules et dendrites de wustite, liées au corroyage par replis successifs (entre 14 et $16 \%$ de la surface analysée du métal), ainsi que des lignes de grains de silice matérialisant des soudures (Fig. 6c). Cette barre est composée de feuilles d'acier à moins de $0,1 \%$ de carbone et de feuilles d'acier eutectoïde $(0,7 \% \mathrm{C})$. La soie de préhension, elle aussi sectionnée à chaud, indique une propreté inclusionnaire équivalente à l'échantillon précédent, bien que les indices de corroyage soient plus faibles (entre 12 et $13 \%$ 

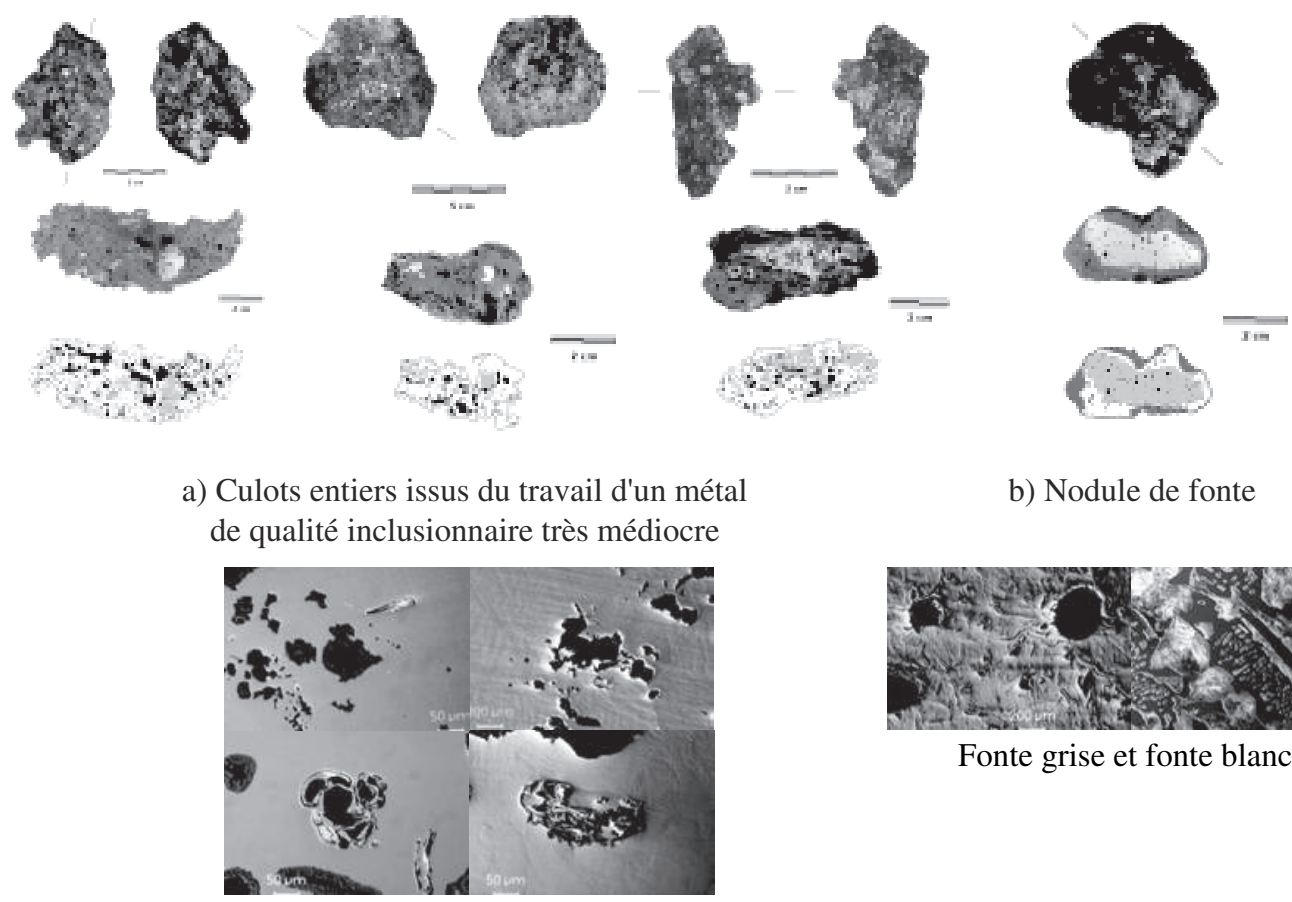

b) Nodule de fonte

Porosités et replis provenant des chutes métalliques

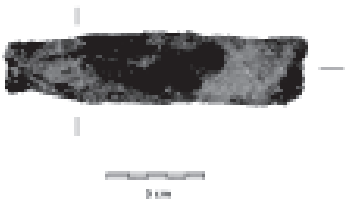

c) Barre à douille sectionnée à chaud

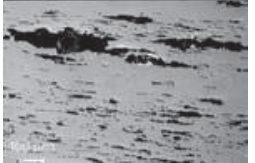

Nombreuses lignes de porosités issues du corroyage et inclusions de silice le long díune soudure

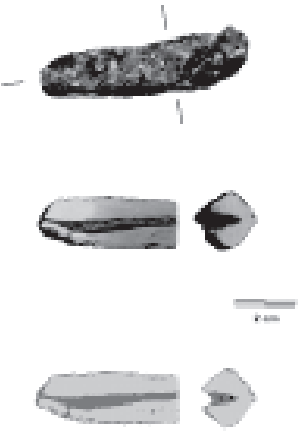

d) Soie de préhension issue du repli d'une barre à douille

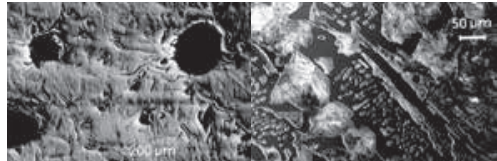

Fonte grise et fonte blanche
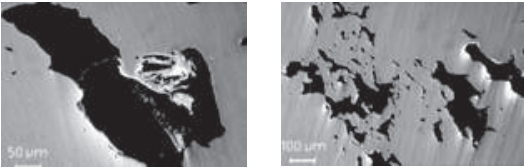

Nombreuses porosités

Figure 6: Récapitulatif des analyses du site de Condé-sur-Suippe «La Sucrerie » (Aisne).

Figure 6: Summary of the analysis of the Condé-sur-Suippes "La Sucrerie" site (Aisne).

d'impuretés sur la surface analysée du métal) (Fig. 6d). Les inclusions sont fayalitique avec de très fines dendrites de wustite. Sa composition est hétérogène, allant d'un acier à $0,1 \%$ de $\mathrm{C}$ en périphérie, à un acier proche de l'eutectoïde à $0,6 / 0,7 \% \mathrm{C}$ plus on se rapproche de la zone repliée.
Les sept autres culots analysés ne renferment aucune perte de fer métallique et semblent donc plutôt correspondre à un travail plus en aval de la chaîne opératoire, à partir d'un métal déjà mieux compacté. 
Ainsi, le métal semble, au moins en partie, arriver sur le site sous la forme de masses brutes ou quasi brutes. Celles-ci sont alors transformées soit en demi-produits mieux compactés, soit directement en produits finis sans passer par un produit intermédiaire.

\subsection{Ronchères « le Bois de la forge "}

Le site de Ronchères « le Bois de la Forge " (Aisne) est localisé à une vingtaine de kilomètres au nord-est de ChâteauThierry (Malrain 2003; Malrain et al. 2004; Bauvais et al. à paraître). Il est implanté sur un plateau qui culmine à 226 m, où l'Ourcq prend sa source. Les deux hectares décapés ont permis la reconnaissance d'un enclos trapézoïdal d'une surface d'un peu moins de 1 hectare.

L'occupation est principalement datée de la Tène D1 et $\mathrm{D} 2$ avec un prolongement jusqu'au $\mathrm{II}^{\mathrm{e}}$ siècle de notre ère. La position stratégique du site, les fossés imposants, la pratique de banquets, et la qualité de la vaisselle confèrent à cette occupation un caractère aristocratique.

Les résultats d'analyse :

Cette installation a livré 12,6 kg de déchets scoriacés, dont trente culots de forge entiers. Dix d'entre eux ont été étudiés. Le milieu d'enfouissement a particulièrement hydroxydé les fragments de métal que ces culots renfermaient, car la majorité de ceux-ci a été rejetée dans le fossé d'enceinte en eau. Pour les mêmes raisons de conservation, l'état du mobilier métallique n'a pas permis de mettre en évidence d'éventuels déchets ou chutes liés à cette activité de forge.

Les fragments métalliques perdus dans le foyer lors des activités de forge attestent du travail d'un métal de qualité inclusionnaire moyenne.

Deux culots analysés, qui sont représentatifs d'environ $60 \%$ du corpus, contiennent de très nombreuses pertes métalliques sous la forme de gros fragments et de quelques chapelets et semis entièrement hydroxydés, et seuls des fantômes d'aiguilles de ferrite aciculaire nous permettent d'identifier un acier très fortement carburé, proche de l'eutectoïde (entre 0,4 et $0,8 \%$ de carbone) (Fig. 7a). Ces pertes représentent entre 6 et $22 \%$ de la surface de la coupe.

L'hydroxydation empêche de caractériser la propreté inclusionnaire du métal, toutefois, les contours très irréguliers des fragments et la très forte proportion de battitures globulaires dans ces échantillons les rapprocheraient d'un travail de demi-produit de qualité inclusionnaire moyen.

Un échantillon présente quelques divergences avec les culots précédents (Fig. 7b). Il ne présente pas d'hydroxydation interne, contrairement au reste de l'ensemble, et nous permet de caractériser plus précisément sa propreté inclusionnaire. Il se caractérise par une très forte proportion de perte de métal sous la forme de gros fragments et de fragments épars particulièrement hétérogènes. Il renferme à la fois des fragments de très bonne propreté inclusionnaire (autour de $2 \%$ d'impureté), avec une morphologie régulière et parfois des restes de plans de frappes encore visibles mais aussi des fragments très irréguliers comportant de nombreuses inclusions de scorie originelle, ainsi que des replis plus ou moins vidés de leur scorie (de 4 à $13 \%$ d'impureté). Leur composition est également très disparate, il peut en effet s'agir de fer doux à moins de 0,1\% de carbone ou d'acier à $0,3 \%$.

Le métal arrivant sur ce site semble posséder une propreté inclusionnaire moyenne, au moins pour une partie des demi-produits importés. Toutefois, la mauvaise conservation des déchets empêche de l'affirmer avec certitude.

\subsection{Braine « la Grange des Moines »}

Le site de Braine est localisé dans la vallée de la Vesle à quelques $\mathrm{km}$ de sa confluence avec l'Aisne.

Ce site, daté de La Tène D1b à La Tène D2b, se compose de deux enclos successifs. Au cours de l'occupation du second enclos, la vocation du site semble changer pour passer d'une ferme aristocratique à un enclos cultuel, avec de nombreux dépôts dans les fossés et des indices de pratique de banquet déjà décelables dans le premier état de l'occupation (Auxiette et al. 1997; Auxiette et al. 2000).

Les résultats d'analyse :

Les fouilles de ce site ont livré $3,06 \mathrm{~kg}$ de scorie dont 13 culots entiers. Huit culots de ce corpus ont été analysés. Parmi les objets métalliques, 6 barres à douille ont également été retrouvées ainsi que 2 soies de préhension. Le degré d'hydroxydation de ces barres n'a permis d'en analyser qu'une seule de façon exhaustive. Les restes métalliques non corrodés des autres demi-produits attestent d'une structure identique à celle-ci.

Les chutes de métal découvertes à l'intérieur de 2 culots analysés sont caractéristiques d'un métal de qualité inclusionnaire moyenne (Fig. 8a). Ils présentent une forte proportion de pertes de métal sous la forme de fragments d'acier de 0,6 à $0,8 \%$ de $\mathrm{C}$ (entre 4 et $6 \%$ de la surface de la coupe). Ces fragments ont des contours déchiquetés et comportent de nombreuses inclusions fayalitiques et amorphes, ainsi que des porosités (environ $14 \%$ d'impureté). Ces culots révèlent également un grand nombre de battitures billes et globulaires 

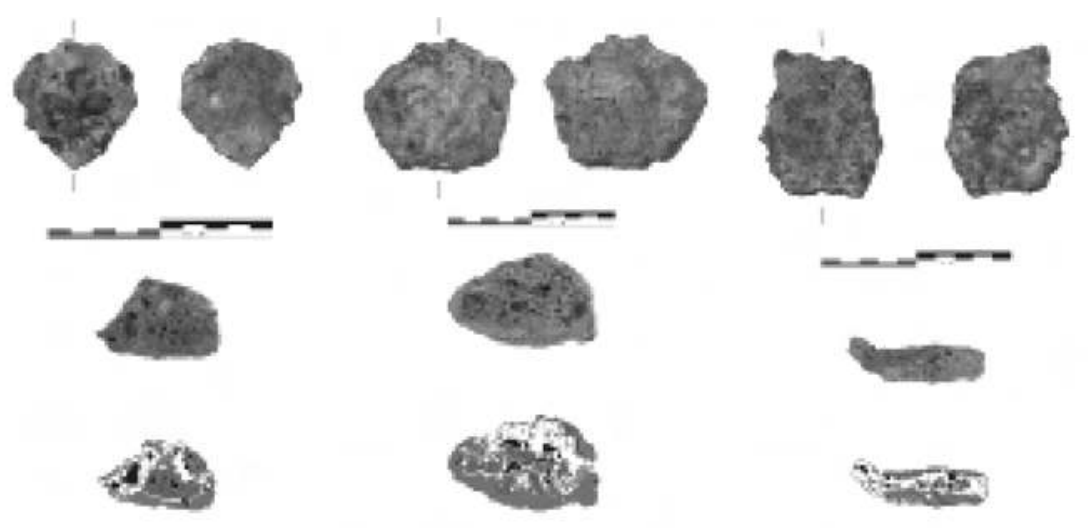

a) Culots fortement hydroxydés issus du travail de métal de mauvaise qualité inclusionnaire

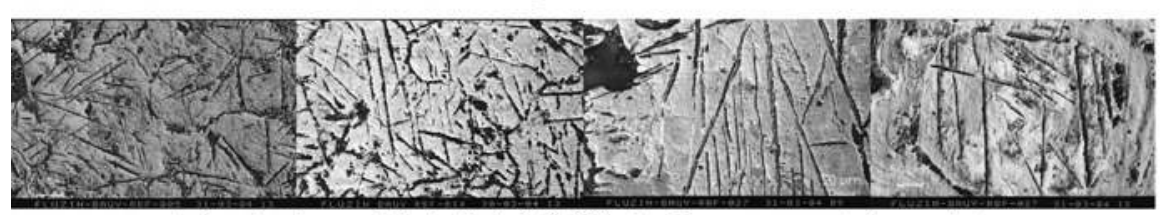

Acier hydroxydé de 0,4 à $0,8 \%$ de $\mathrm{C}$ provenant des culots

Seules les fantômes d'aiguilles de ferrite sont visibles

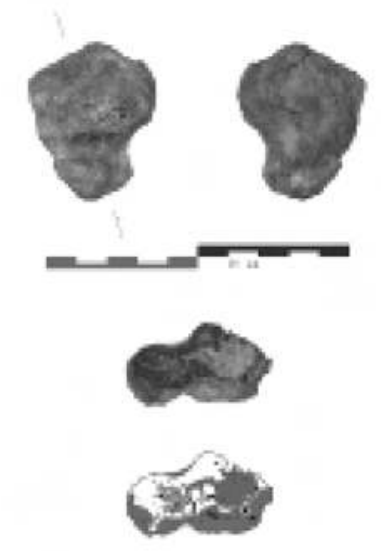

b) Culot très hétérogène

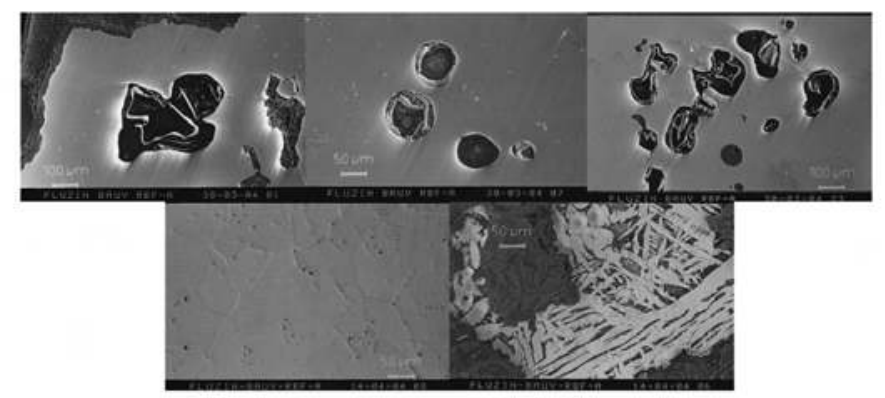

Hétérogénéité de structure et de composition

Figure 7 : Récapitulatif des analyses du site de Ronchères « Le Bois de la Forge » (Aisne).

Figure 7: Summary of the analysis of the Ronchères "Le Bois de la Forge" site (Aisne). 
ainsi que quelques battitures plates, ce qui va dans le sens d'une succession de phases de travail de natures différentes.

L'une des soies comporte de nombreuses inclusions linéaires de scorie fayalitique avec de très fines dendrites de wustite, dont la proportion est équivalente à celle retrouvée dans le métal des culots (13 à $16 \%$ d'impureté) (Fig. 8b). Toutefois, sa composition entièrement ferritique ne permet pas de la rattacher aux chutes d'acier présentes dans ceux-ci.

Le reste des objets métalliques liés aux activités de forge atteste d'un métal de bonne qualité inclusionnaire. Les « barres à douille " sectionnées à chaud de cet atelier présentent des lignes d'inclusions d'amorphe et de scorie fayalitique à très gros globules de wustite, ainsi que des porosités déformées par le corroyage, mais dans une proportion relativement faible (de 2 à $4 \%$ d'impureté) (Fig. 8c). Tout au long de la barre analysée, majoritairement composée de ferrite à gros grains, on peut suivre une ligne de soudure dont les abords présentent une composition plus aciérée $(0,2$ à $0,3 \% \mathrm{C}$ ).

La seconde soie, qui se compose d'un repli de feuilles métalliques avec des lignes plus aciérées au niveau des soudures, peut résulter d'un repli sur elle-même d'une de ces barres à douille (Fig. $8 \mathrm{~d}$ ). Elle présente également une très bonne propreté inclusionnaire (de 1,5 à $2 \%$ d'impureté) qui s'avère même meilleure, ce qui semble logique, s'il y a eu une nouvelle phase de martelage liée à sa mise en forme.

Globalement, le métal travaillé sur le site semble posséder une bonne propreté inclusionnaire. Toutefois, certaines pertes contenues dans les culots laissent envisager que le métal initialement travaillé est d'une qualité inclusionnaire moyenne.

\subsection{Villeneuve-Saint-Germain « les Etomelles »}

Le site est localisé sur la commune de Villeneuve-SaintGermain, jouxtant la ville de Soissons (Hénon à paraitre). Cette installation agricole est seulement éloignée de quelques $\mathrm{km}$ de l'Oppidum du même nom, mais présente un décalage chronologique avec celui-ci. En effet, l'agglomération urbaine est datée de La Tène D2a/D2b alors que l'installation agricole est juste antérieure (La Tène D1b). On peut imaginer que la population de ce site est à l'origine d'une partie des occupants de l'oppidum postérieur.

Les résultats d'analyse :

Sur la surface fouillée de ce site, $1,5 \mathrm{~kg}$ de scorie, dont 8 culots de forge entiers, ont été mis au jour. Parmi eux, 4 ont été analysés ainsi que 2 barres à douille complètes et 1 sectionnée à chaud.

L'ensemble des investigations révèle une activité de forge d'élaboration d'objet à partir d'un métal de bonne qualité inclusionnaire. On note tout d'abord de très faibles pertes de métal dans les culots. Toutefois, une scorie magnétique livre des fragments métalliques de tailles plus conséquentes, mais dont la propreté inclusionnaire reste bonne, malgré un taux d'inclusion de $0,5 \%$, légèrement supérieur aux demiproduits analysés (Fig. 9a).

En effet, les barres à douille entières et sectionnées à chaud en contexte d'atelier comportent quelques fines lignes d'inclusions fayalitiques liées à un corroyage de qualité, conférant à ces objets une bonne propreté inclusionnaire (de 1,5 à $3 \%$ d'inclusions). Leur composition est hétérogène, avec une face ferritique à gros grains et une face aciérée à $0,5 \%$ $\mathrm{C}$ visiblement par cémentation (Fig. $9 \mathrm{~b}$ et c).

\subsection{Bazoches-sur-Vesle « les Chantraines»}

Le site de Bazoches-sur-Vesle « les Chantraines » est localisé dans le département de l'Aisne, à quelques $\mathrm{km}$ de sa limite avec la Marne (Pommepuy, Gransar 1998; Pommepuy et al. 2000; Bauvais, Fluzin 2005; Gransar, Pommepuy 2005; Bauvais et al. à paraitre). Son occupation principale peut être datée de la seconde moitié du II ${ }^{\mathrm{e}}$ siècle avant notre ère, entre -150 et -100 environ (La Tène D1a). L'enclos, de morphologie irrégulière, couvre une surface de 1,55 ha, et l'espace interne est décomposé en plusieurs zones par des fossés de partition.

Le statut aristocratique de cet établissement rural est prouvé par sa structuration générale (surface importante et structuration spatiale rigoureuse, dimension des fossés et architecture ostentatoire), mais aussi par son mobilier abondant et riche (forte proportion de céramique tournée, inhabituelle à La Tène D1a, grande abondance d'amphores gréco-italiques et de monnaies gauloises, présence d'armement et d'un denier républicain en argent, indices matériels de pratique de banquets et présence de dépôts symboliques dans les fossés).

Les résultats d'analyse :

Le site a livré relativement peu de déchets sidérurgiques avec $1,15 \mathrm{~kg}$ de scorie dont 6 culots entiers. Cinq de ces culots ont été analysés. La fouille a également révélé 10 fragments de demi-produits de type barre à douille.

Les demi-produits issus de ce site n'ont pas pu faire l'objet d'investigations métallographiques, toutefois ils peuvent être, par extension, considérés comme les échantillons des 


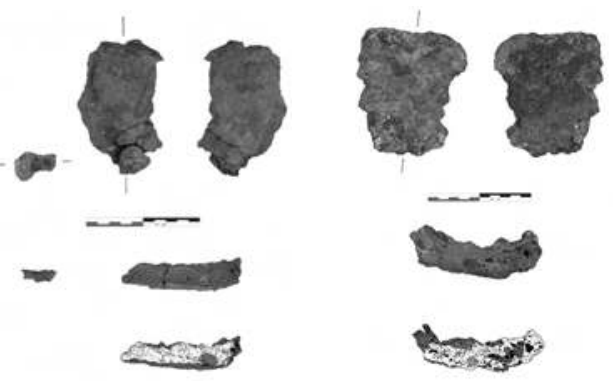

a) Culots issus du travail d'un métal de mauvaise qualité inclusionnaire

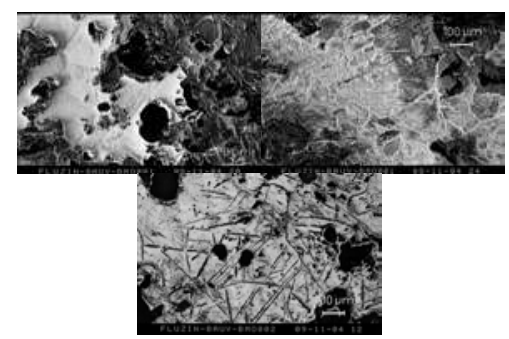

Acier de propreté inclusionnaire médiocre provenant des culots

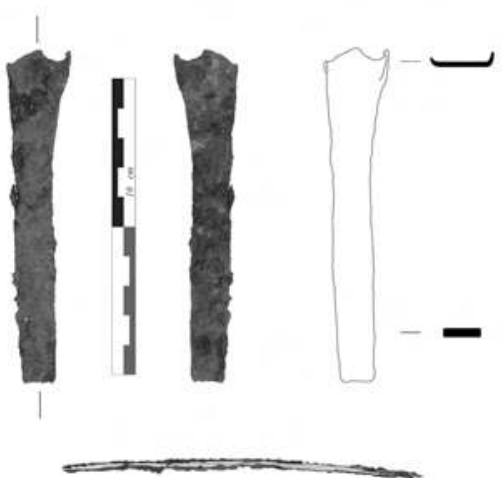

c) Barre à douille sectionnée à chaud de bonne qualité inclusionnaire

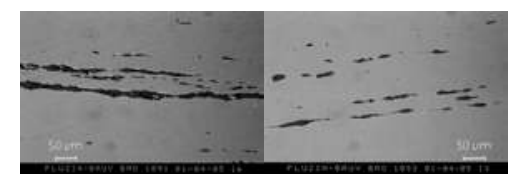

Fines lignes d'inclusions issues du corroyage

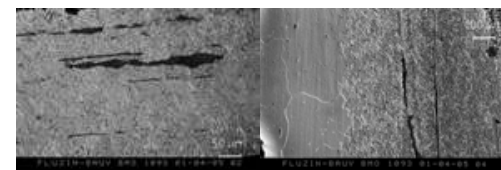

Composition hétérogène de ferrite et díacier de 0,1 à $0,4 \%$ C

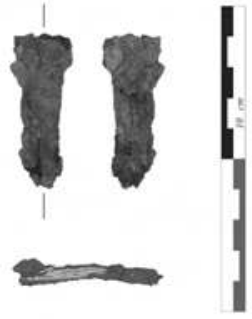

b) Soie de préhension de mauvaise qualité inclusionnaire

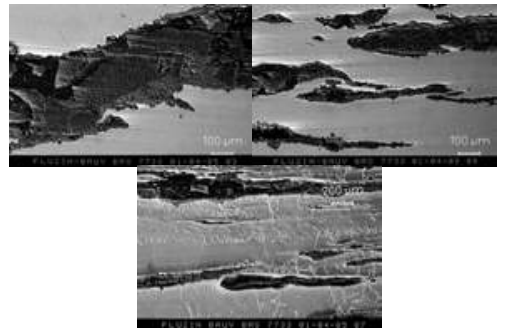

Lignes de corroyage avec inclusions de scorie dans un métal entièrement ferritique

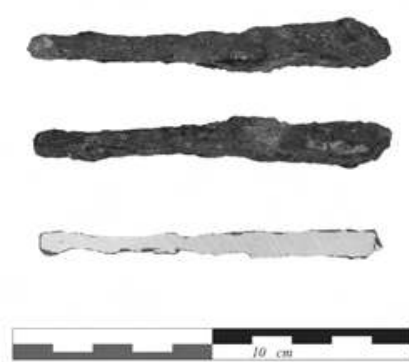

d) Soie de préhension de bonne qualité inclusionnaire

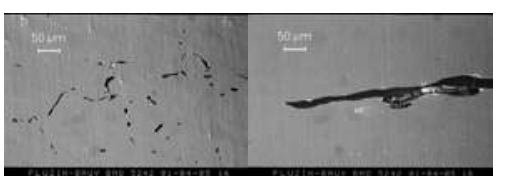

Rares inclusions et porosités

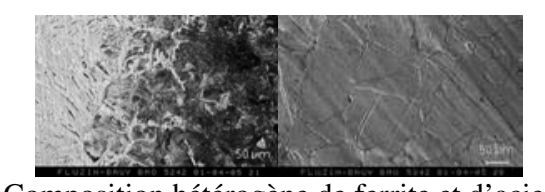

Composition hétérogène de ferrite et d'acier de 0,2 à $0,7 \% \mathrm{C}$

Figure 8 : Récapitulatif des analyses du site de Braine «La Grange des Moines » (Aisne). Figure 8: Summary of the analysis of the Braine "La Grange des Moines" site (Aisne). 

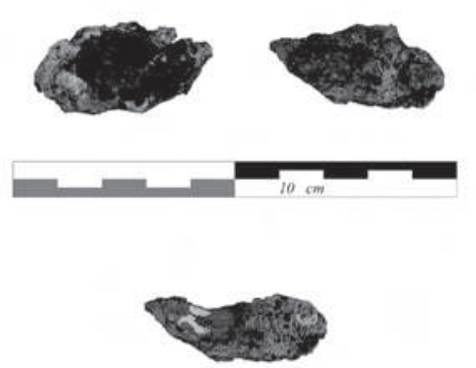

a) Scorie magnétique

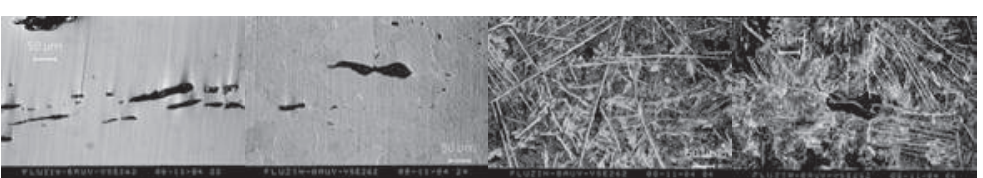

Lignes d'inclusions liées au corrayage dans un métal hétérogène composé de ferrite à $0,02 \% \mathrm{C}$ et d'acier à 0,6 - 0,7\% C
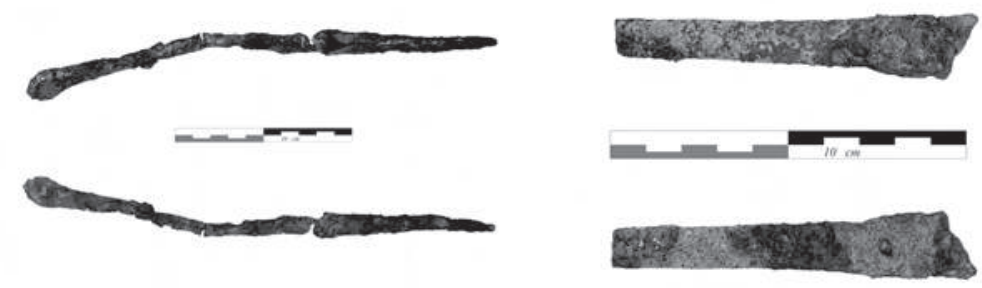

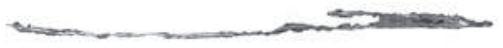

b) Barre à douille entière

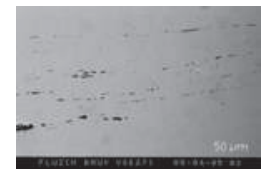

Rares inclusions issues du corroyage

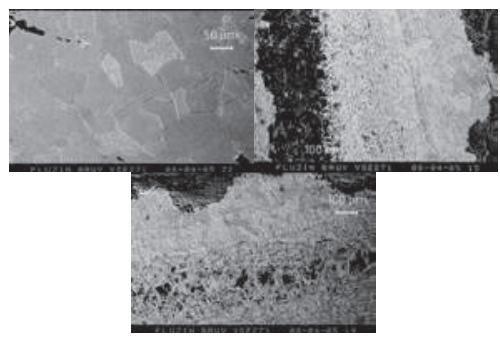

Composition hétérogène de ferrite et díacier de 0,1 à $0,7 \% \mathrm{C}$ avec phénomène de feuilleté (cémentation?)

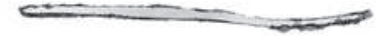

c) Barre à douille sectionnée à chaud

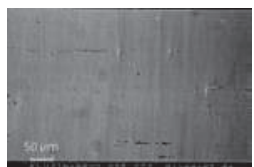

Rares inclusions issues du corroyage

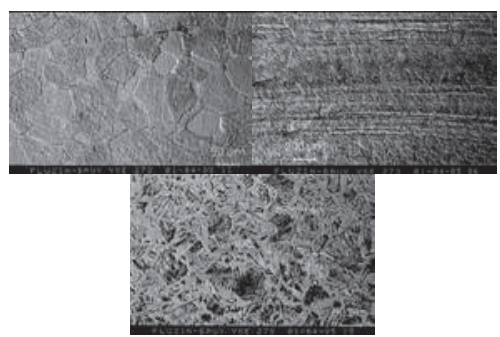

Composition hétérogène de ferrite et d'acier de 0,1 à $0,4 \% \mathrm{C}$

Figure 9 : Récapitulatif des analyses du site de Villeneuve-Saint-Germain «Les Etomelles" (Aisne).

Figure 9: Summary of the analysis of the Villeneuve-Saint-Germain "Les Etomelles" site (Aisne). 
autres sites étudiés, comme des demi-produits de bonne qualité inclusionnaire.

Les culots analysés vont dans ce sens, avec une absence complète de pertes de métal, hormis quelques petites billes et chapelets métalliques.

\section{RÉFLEXIONS SUR L'ORGANISATION TECHNIQUE DE LA POST-RÉDUCTION ET SES IMPLICATIONS SOCIALES :}

\subsection{Les différents états de la matière}

Suite à ces analyses, il est possible de classer schématiquement les demi-produits selon leur homogénéité :

La qualité inclusionnaire du métal présent sur ces sites peut se diviser en 3 catégories :

- Une qualité mauvaise proche de la masse brute, caractérisée par un aspect déchiqueté en périphérie et par un taux d'impureté d'environ 15 à $25 \%$ (Condé-sur-Suippe);

- Une qualité moyenne, caractérisée par un taux d'impureté d'environ 10 à $15 \%$ (Condé-sur-Suippe, Ronchères, Braine);

- Une bonne qualité avec un taux d'impureté de 0 à 10 \% (Braine, Villeneuve-Saint-Germain «les Etomelles ", Bazoches-sur-Vesle)

Leur composition fer-acier est majoritairement composite de 0,02 à $0,7 \% \mathrm{C}$ et provient à la fois de l'hétérogénéité initiale des masses travaillées (corroyage), de soudures, et de probables cémentations périphériques.

\subsection{Discussion}

À partir de ces constats, trois questions se posent :

1- Les différences qualitatives sont-elles associées à un type de production particulier?

Dans le cas des barres à douille, leur alliage composite de fer et d'acier, leur taille ainsi que leur bonne propreté inclusionnaire en font des demi-produits ou des ébauches plutôt destinés à de petits objets (parure, instruments de toilette...) ou à des produits plats (lames de couteau, bandes de tôle...). Toutefois, il est envisageable que par repli et soudure d'une ou plusieurs barres, il soit possible d'élaborer des objets de taille plus conséquente. Les barres sont tout de même destinées à des objets réclamant une grande qualité de métal.

En revanche, la quantité et la taille des fragments d'acier des culots de Braine et de Ronchères indiquent que les demiproduits travaillés sont de tailles plus conséquentes que ceux précédemment cités. Leur forte teneur en carbone de 0,6 à $0,7 \%$ révèle également d'importantes différences.

Les variations de tailles, d'états inclusionnaires et de compositions incitent à voir, dans ces demi-produits, des vocations différentes, surtout lorsqu'on recense plusieurs d'entre eux sur un même site. Cela exclut en partie d'éventuelles différences liées à des fluctuations d'approvisionnement.

La présence d'une mauvaise qualité de métal sur le site de Condé-sur-Suippe, qui possède le rang hiérarchique le plus élevé, à l'intérieur de son contexte régional, ne semble pas non plus être liée à un problème d'accès au réseau d'acquisition de la matière première. Elle peut ainsi correspondre à une vocation de production particulière. Toutefois, les activités de ce site semblent, au moins en partie, destinée à une fabrication d'objets nécessitant une excellente qualité de métal (tôle). Ainsi, elle apparaît comme étant davantage liée à une phase technique de mise en forme de la matière plus ou moins brute en demi-produit avant transformation finale.

Il est ici intéressant de remarquer que la quantité de déchets scoriacés retrouvés sur chacun des sites diminue proportionnellement à l'accroissement de la qualité du métal travaillé (Fig. 10). Deux facteurs semblent se «télescoper ». En effet, lors de l'élaboration d'un même objet, plus le métal est de bonne qualité inclusionnaire, plus la quantité de déchet produite sera faible. L'intensité de l'activité d'un site et la durée de son occupation devraient changer cet état de fait; il n'en est rien. Faut-il y voir également un rapport entre la qualité du métal acquis par un site et l'intensité de sa production? D'autant que l'on sait que le site de Condésur-Suippe possède la durée d'occupation la plus courte, la quantité de scorie la plus importante et la qualité de métal la plus mauvaise.

Il faut tout de même relativiser cette comparaison sachant que la quantité de déchets engendrée par les activités de ces sites reste faible à côté de celle d'autres régions et surtout de celle de la période gallo-romaine qui suit.

2- Ces variations qualitatives sont-elles liées à la capacité économique des artisans opérant sur les différents sites?

La réponse est ici difficile dans l'état actuel des recherches. Seuls les sites de rang hiérarchique élevé, et donc qui possèdent un pouvoir économique important, révèlent des activités d'artisanat sidérurgique, contrairement aux petites activités d'entretien domestique présentes sur la majorité des installations agricoles.

La présence d'un métal de mauvaise qualité sur l'oppidum de Condé-sur-Suippe, qui représente le rang hiérarchique 


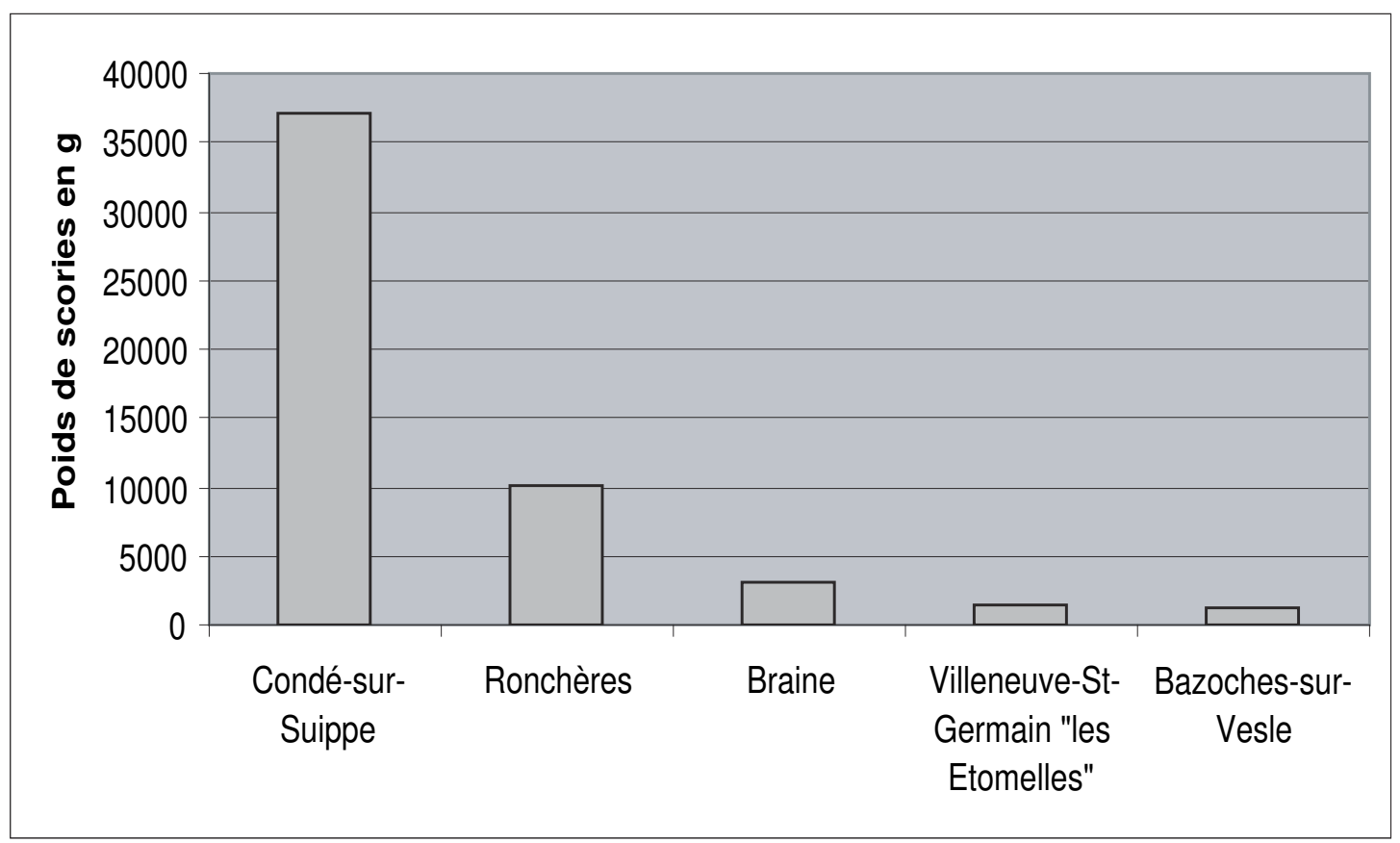

Figure 10 : Histogramme des poids de scories par sites étudiés.

Figure 10: Histogram of the slags weights by studied sites.

le plus élevé, ne semble pas aller dans le sens d'une répartition des demi-produits selon la capacité économique des sites. Toutefois, le caractère très particulier de ces sites protourbains, avec une concentration artisanale importante et qui regroupe une population hétérogène ne répond peut-être pas aux mêmes stratégies économiques que les autres sites d'atelier (Bauvais 2001).

3- Ces différences qualitatives sont-elles reliées à une organisation de l'acquisition/redistribution du métal dans un contexte d'approvisionnement à moyenne ou longue distance?

Nous nous heurtons ici à un problème de chronologie :

À La Tène D, apparaissent les premiers oppida. Ces sites concentrent une grande quantité d'artisanats en tous genres et semblent centraliser un grand nombre d'activités et de biens. Il s'agit des prémices d'un pouvoir centralisé. L'oppidum de Condé-sur-Suippe, ainsi que celui de Villeneuve-Saint-Germain (Bauvais à paraître) mais aussi de Bibracte et d'Entremont (Travaux Marion Berranger en cours) recèlent justement cette matière première très médiocre. Est-ce le signe d'une centralisation dans l'acquisition de la matière dans un contexte d'approvisionnement à moyenne ou longue distance? Cela semble contradictoire avec les phénomènes de diffusions déjà mis en évidence à d'autres périodes pour d'autres objets et matériaux. En effet, ce sont les produits à forte valeur ajoutée qui ont tendance à se diffuser en plus grand nombre et à longue distance (lames de silex du Grand-Pressigny; obsidienne de l'Anatolie; pâte de verre italique; vaisselle en bronze grecque et romaine...). Or, les sites qui acquièrent cette matière, via vraisemblablement un réseau d'échange à longue distance, le feraient sous sa forme la plus brute. Dans ce cas, sommes-nous réellement dans un cas de figure d'absence de production locale de fer?

Quoi qu'il en soit, ces sites revêtiraient un statut de centres redistributeurs. Les barres à douilles, qui semblent se localiser uniquement dans le nord du Bassin parisien et être les demiproduits les plus diffusés dans la région, apparaissent avant la formation des oppida. Les sites ont-ils repris à leur compte cette tradition d'approvisionnement, voire cette production?

Dans ce contexte, il serait possible de mettre en évidence trois niveaux d'intégration des sites dans le réseau d'acquisition/redistribution (Fig. 11) :

- Niveau 1, les sites importateurs de matière sous forme de demi-produits plus ou moins bruts et qui les transforment (demi-produits, objets); Condé-sur-Suippe et peutêtre Ronchères.

- Niveau 2, les sites qui acquièrent la matière sous forme de demi-produits transformés pour une élaboration d'objet. À l'intérieur de ce niveau, deux catégories se distinguent suivant la qualité inclusionnaire du métal acquis; Ronchères, Braine, Villeneuve-Saint-Germain "Les Etomelles ».

- Niveau 3, les sites consommateurs d'objets en fer. 


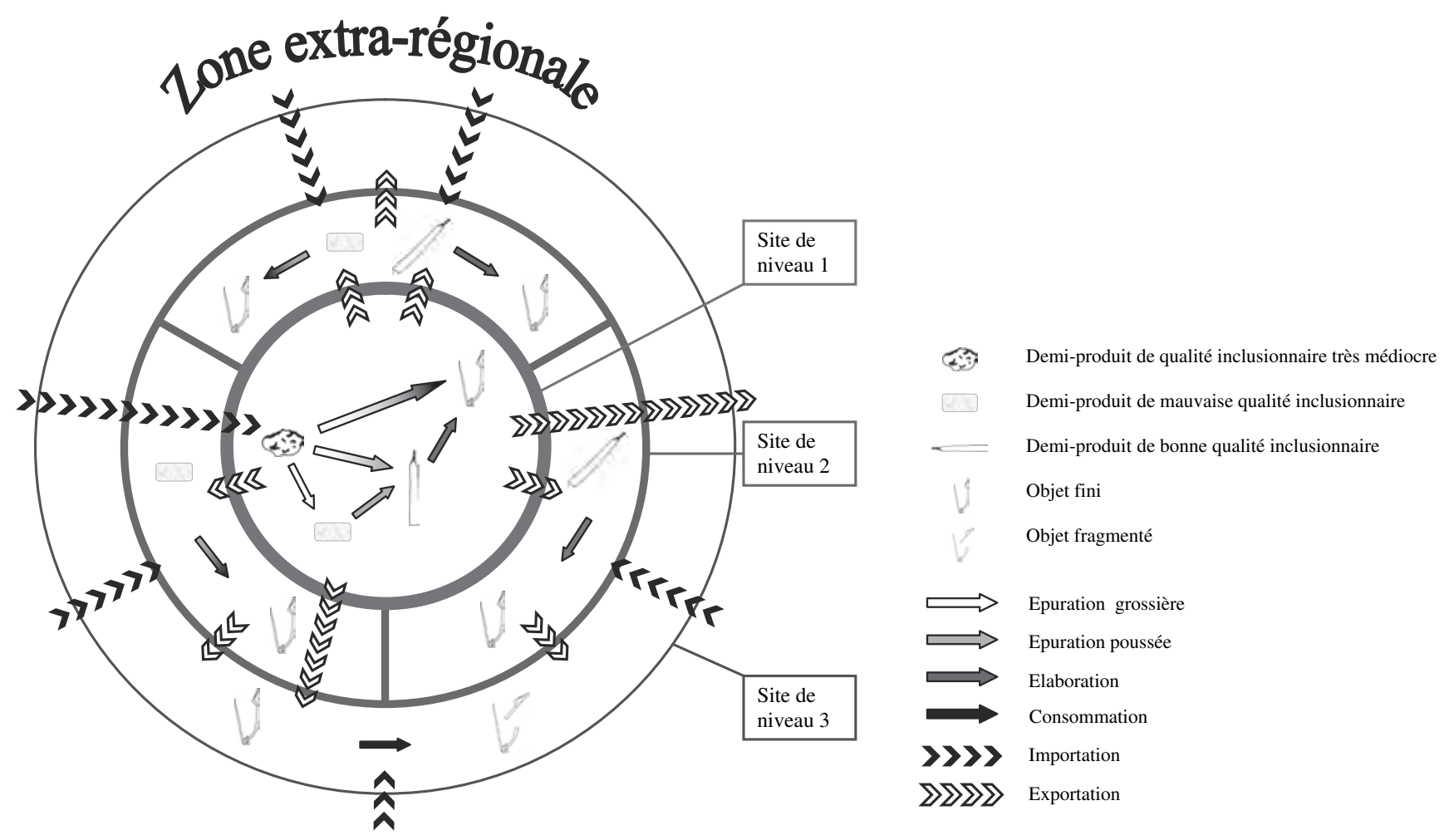

Figure 11 : Représentation schématique des différents niveaux d'intégration des sites de l'Aisne à La Tène finale dans le réseau d'acquisition/redistribution des matières premières ferreuses.

Figure 11: Simplistic representation of the various integration levels of the final La Tène Aisne sites in the acquisition/redistribution network of the ferrous raw materials.

Dans l'état actuel des recherches, aucun site indiquant une production importante, ou recelant des indices d'importation de demi-produits d'épuration très médiocres n'est connu avant La Tène D1b, c'est-à-dire avant l'apparition des oppida.

\section{Conclusion}

Les résultats acquis jusqu'à présent permettent d'entrevoir une organisation complexe des activités de forge dont les principaux modes nous restent encore majoritairement inconnus.

On remarque dans cette région, en ce qui concerne les activités sidérurgiques, un croisement constant entre des impératifs d'ordres géographiques (absence de réduction), technologiques (morcellement de la chaîne opératoire et typologie spécifique des demi-produits) et socio-économiques (organisation de la société).

La prise en compte globale de ces différents facteurs alliée à une multiplication des études de sites (15 sites seront analysés dans l'Aisne et ses environs) permettront, à terme, d'avoir une vision plus approfondie de cette organisation. Une recherche plus poussée devra également être mise en œuvre pour les périodes antérieures à l'apparition des oppida, afin de cerner les changements propres à la métallurgie du fer dans ce contexte de mutation sociopolitique.

Une campagne de caractérisation micro-analytique des inclusions dans le métal est en cours au sein du LRC (laboratoire commun LPS CEA - Saclay et laboratoire Métallurgies et Cultures de l'UMR 5060 du CNRS), afin de rechercher des critères de composition discriminants et de discerner d'éventuelles différences dans l'origine de ces demi-produits.

\section{Bibliographie}

Anderson, T. J., Augustoni, C., Duvauchelle, A., Serneels, V. et Castella, D., 2003. Des artisans à la campagne. Carrière de meules, forge et voie romaine à Châbles (FR), Academic Press Fribourg, Fribourg, 390 p. (Archéologie Fribourgeoise 19). 
Auxiette, G., Desenne, S., Gransar et F., Pommepuy, C., 1997. Le site de Braine "la Grange des Moines". Document Final de Synthèse, SRA Picardie, AFAN Antenne Nord-Picardie.

Auxiette, G., Desenne, S., Gransar, F. et Pommepuy, C., 2000. Structuration générale du site de Braine "la Grange des Moines » (Aisne) à La Tène finale et particularités : présentation préliminaires, in Les enclos pourquoi faire?, actes de la table ronde de Ribemont-sur-Ancre, Revue archéologique de Picardie, $\mathrm{n}^{\circ} 1 / 2,2000$, p. 97-104.

Bauvais, S., 2000. Analyse de la Chaîne-Opératoire de sidérurgie sur l'oppidum de Condé-sur-Suippe (Aisne), Mémoire de maîtrise, Université de Paris I Panthéon-Sorbonne, France.

BauvaIs, S., 2001. La sidérurgie dans la civilisation des oppida: approches théoriques et état des connaissances, Mémoire de DEA, Université de Paris I Panthéon-Sorbonne, France.

Bauvais, S. et Fuuzin, P., 2005. Analyses métallographiques des déchets de forge du site de Bazoches-sur-Vesle "les Chantraines" : confrontation et perspective régionale (Aisne), in G. Auxiette, F. Malrain, S. Desenne et B. Robert (dir.), Hommage à Claudine Pommepuy. Revue Archéologique de Picardie (Numéro spécial 22).

Bauvais, S., Gaudefroy, S., Gransar, F., Malrain, F. et Fluzin, P., sous presse. Premières réflexions sur l'organisation des activités de forge en contexte rural à La Tène finale en Picardie, Actes du XXVIII colloque international de l'AFEAF, Toulouse 20-23 mai 2004, Aquitania.

Bauvais, S. et Pion, P., sous presse. Analyse de la chaîne opératoire de la sidérurgie sur l'oppidum de Condé-sur-Suippe, Gallia.

Crew, P., 1991. The experimental production of prehistoric bar iron, Historical Metallurgy, 25, $\mathrm{n}^{\circ} 12$, p. 21-36.

Daveau, I. et Goustard, V., 2000. Un complexe métallurgique et minier du Haut Moyen Âge. Le site des Fourneaux à VertSaint-Denis (Seine-et-Marne), Gallia 57, CNRS, p. 1-158.

Dillmann, P., Fluzin, P. et Benoit, P., 1998. Du fer à la fonte. Nouvelles approches archéométriques, in Linnovation technique au Moyen Âge: actes du VI ${ }^{\mathrm{e}}$ Congrès de la société d'archéologie médiévale, Dijon, octobre 1996, Paris, Errance, p. $160-168$.

Duniкоwsкi, C., 1995. Approche méthodologique d'un ferrier : l'exemple du site des Clérimois, In P. Benoit et P. Fluzin (dir.), Paléométallurgie du fer et cultures, Actes du Symposium international du Comité pour la Sidérurgie Ancienne, Belfort - Sévenans, 1-2-3 novembre 1990, Paris, Éditions Vulcain/ AEDEH, p. 325-332.

Duniкоwsкi, C. et CAввоI, S., 1995. La sidérurgie chez les Sénons : les ateliers celtiques et gallo-romains des Clérimois (Yonne), Paris, Éditions de la Maison des Sciences de l'Homme, coll. "Documents d'Archéologie Française; 51 », 186 p.
Dunikowski, C. et Cabboi, S., 2001. L'extraction du minerai de fer, la production du charbon de bois et la réduction du minerai de l'Âge du fer, de la période romaine et du haut Moyen Âge dans le nord de la France (les exemples du site des Clérimois, département de l'Yonne et ensemble des sites de la Sarthe), in L'obtentcio del ferro pel procediment directe entre els segles $I V i$ $X I X$, Actes del Gé curs d'arqueologia d'Andorra, Andorre, $2 \mathrm{au}$ 5 octobre 2000, Area de Recerca Historica, p.189-210.

Gransar, F. et Pommepuy, C., 2005. Bazoches-sur-Vesle les Chantraines (Aisne) : présentation préliminaire de l'établissement rural aristocratique de La Tène D1, in G. Auxiette, M. Malrain, S. Desenne, B. Robert (dir.). Hommage à Claudine Pommepuy, No hors série de la Revue Archéologique de Picardie (Numéro spécial 22).

Fuuzin, P., 1999. Ponte di val Gabbia III (Bienno) i reperti metallici dalla forgia. Primi risultati dello studio metallografico, in C. Cucini Tizzoni et M. Tizzoni (dir.), La minera perduta. Cinque anni di recerche archeometallurgique nel territorio di Bienno, Bienno, Breno, p. 61-92 et 189-194.

FLuzin, P., 2002. La chaîne-opératoire en sidérurgie : matériaux archéologiques et procédés. Apport des études métallographiques, in $\mathrm{H}$. Bocoum (dir.), Aux origines de la métallurgie du fer en Afrique. Une ancienneté méconnue, Paris, UNESCO, coll. "Mémoire des peuples », p. 59-91.

Fluzin, P., Ploquin, A. et Dabosi, F., 2004. Approches métallurgiques et archéométriques, in M. Mangin (dir.), Le Fer, Paris, Errance, coll. «Archéologique ", p. 113-173.

Hénon, B., à paraître. Le site de Villeneuve-Saint-Germain "les Etomelles». Document Final de Synthèse, SRA Picardie, INRAP Direction Interrégionale Nord-Picardie.

Leroy, M., Merluzzo, P., Fluzin, P., Leclerc, D., Aubert, M. et Ploquin, A., 2000. La restitution du savoir faire pour comprendre un procédé technique : l'apport de l'expérimentation en archéologie du fer, in P. Pétrequin, P. Fluzin, J. Thiriot, P. Benoit (dir.), Arts du Feu et Productions Artisanales, Actes des XX' Rencontres Internationales d'Archéologie et d'Histoire d'Antibes, 21-23 octobre 1999. Antibes, APDCA, p. 37-53.

Lorcquet, P., à paraître. Le site de Roissy-en-Brie "la Ferme Sassinot". Document Final de Synthèse, SRA Île-de-France, INRAP Direction Interrégionale Centre-Ile-de-France.

Orengo, L., 2003. Forges et forgerons dans les habitats laténiens de la Grande Limagne d'Auvergne : fabrication et consommation de produits manufacturés en fer en Gaule à l'Âge du fer, Montagnac Éditions Monique Mergoil, coll. "Monographies instrumentum; 26 », $325 \mathrm{p}$.

Malrain, F., 2003. Le site de La Tène finale de Ronchères « le Bois des Forges" (Aisne), in F. Malrain, C. Jouannet (dir.), Bulletin de l'Association Française pour l'Étude de l'âge du Fer, p. 34-35

Malrain, F., Bauvais, S., Boulen, M., Fechner, K., Henon, B., Legros, V., Saurel, M. et Pissot, V., 2004. Ronchères (Aisne) 
"le Bois de la Forge": site artisanal de La Tène finale et du Gallo-Romain. Document final de synthèse, SRA Picardie, 165 p, 112 fig.

Mangin, M., Courtaudon, J.-L., Fluzin, P. et de Laclos, E., 2000a. Village, forges et parcellaire aux sources de la Seine. L'agglomération antique de Blessey-Salmaise (Côte-d'Or), Besançon, Presses universitaire franc-comtoises, 517 p. (Annales littéraires de l'Université de Franche-Comté; vol. 699).

Mangin, M., Fluzin, P., Courtaudon, J.-L. et Fontaine, M.J., 2000b. Forgerons et paysans des campagnes d'Alésia. Haut Auxois (Côte-d'Or), Ir siècle av. J.-C.-VIIt' siècle apr. J.-C., Paris, CNRS, 508 p.

NoseK, E.-M., 1994. The Metallography of gromps, in M. Mangin (dir.), La sidérurgie ancienne de l'est de la France dans son contexte européen, Actes du Colloque de Besançon, Besançon, 10-13 novembre 1993, Les Belles-Lettres, 422 p. (Annales littéraires de l'Université de Besançon, 536).

Pion, P., Pommepuy, C., Auxiette, G., Hénon, B. et Gransar, F., 1997. L'oppidum de Condé-sur-Suippe/Variscourt (Aisne) (fin II $^{\mathrm{e}}$-début $\mathrm{I}^{\mathrm{er}}$ siècle av. J.-C.). Approche préliminaire de l'organisation fonctionnelle d'un quartier artisanal, in G. Auxiette, L. Hachem et B. Robert (dir.), Espaces physiques espaces sociaux dans l'analyse interne des sites du néolithique à l'Âge du Fer, Actes du $119^{\mathrm{e}}$ Congrès National des Sociétés Historiques et Scientifiques, Amiens, octobre 1994, Paris, CTHS, p. 275-309.

Pommepuy, C. et Gransar, F., 1998. Bazoches-sur-Vesle " les Chantraines"(Aisne). Document final de synthèse, SRA Picardie, AFAN Nord-Picardie, 2 vol.

Pommeruy, C., Auxiette, G., Desenne, S., Gransar, F. et Hénon, B., 2000. Des enclos à l'âge du Fer dans la vallée de l'Aisne : le monde des vivants et le monde des morts, in les enclos pourquoi faire? Actes de la table ronde de Ribemont-sur-Ancre, Revue archéologique de Picardie, $\mathrm{n}^{\circ}$ 1/2, p. 197-217.

Woimant, G.-P., 1990. Beauvais, les Aulnes du Canada : viereckschanze ou " enceinte quadrangulaire "? Revue Archéologique de Picardie, n 3/4, 1990, p. 27-93. 